\title{
Sous la direction de Bernard Paillard, Jean-François Simon et Laurent Le Gall - En France rurale : les enquêtes interdisciplinaires depuis les années 1960- Presses universitaires de Rennes 2010, Centre de recherche bretonne et celtique, 396 p., $20 €$
}

\section{Monique Selim}

\section{(2) OpenEdition}

Édition électronique

URL : http://journals.openedition.org/economierurale/3053

DOI : 10.4000/economierurale.3053

ISSN : 2105-2581

\section{Éditeur}

Société Française d'Économie Rurale (SFER)

\section{Édition imprimée}

Date de publication : 23 mai 2011

Pagination : 93-95

ISSN : 0013-0559

\section{Référence électronique}

Monique Selim, « Sous la direction de Bernard Paillard, Jean-François Simon et Laurent Le Gall - En France rurale : les enquêtes interdisciplinaires depuis les années 1960- Presses universitaires de

Rennes 2010, Centre de recherche bretonne et celtique, 396 p., $20 € »$, Économie rurale [En ligne], 323 | mai-juin 2011, mis en ligne le 23 mai 2011, consulté le 24 septembre 2020. URL : http://

journals.openedition.org/economierurale/3053; DOI : https://doi.org/10.4000/economierurale.3053 
Sous la direction de Bernard PAILLARD,

Jean-François SIMON et Laurent LE GALL

\section{En France rurale Les enquêtes interdisciplinaires depuis les années 1960}

Presses universitaires de Rennes 2010, Centre de recherche bretonne et celtique, 396 p., $20 €$

V oici un livre étonnant, inattendu dans lequel tout chercheur en sciences sociales pourra puiser des éléments de réflexion critique féconds. Cet ouvrage collectif, qui revisite les grandes et petites enquêtes pluridisciplinaires menées en milieu rural français, depuis cinquante ans, est en effet d'une grande portée épistémologique non seulement pour prendre connaissance des pratiques passées d'investigation et des courants disciplinaires qui les sous-tendent mais aussi pour scruter les modalités présentes de recherche au regard de leur passé proche. Si Plozevet si célèbre - occupe la majeure partie des contributions, la confrontation avec l'Aubrac, le Chatillonnais, Minot, les Baronnies, la Corse et Margeride est instructive tout à la fois des permanences disciplinaires, des récurrences topiques mais aussi du surgissement de perspectives originales dues à la nature elle-même des terrains et à leur évolution. Issu d'un colloque qui s'est tenu en Bretagne en 2008 et qui a rassemblé surtout des protagonistes des études effectuées, cette somme se nourrit des regards que ces acteurs singuliers de la recherche jettent sur les débuts de leur professionnalisation et des points de vue qu'ils adoptent sur leur participation à la recherche. L'intérêt principal de l'ouvrage réside là, dans les matériaux personnels et collectifs inédits de ce qui se donne à lire en quelque sorte comme une seconde enquête menée en toute conscience par des chercheurs arrivés au terme de leur carrière, sur leur propre trajectoire intellectuelle.
Les années 60 consacrent la percée scientifique de la ruralité française à travers les enquêtes financées par l'État qui regroupe sociologues, ethnologues, économistes, géographes, historiens, démographes. Pour certaines d'entre elles, des chercheurs en sciences du vivant sont conviés, tels des médecins hématologues dans les Pyrénées. L'acronyme de ces recherches coopératives sur programme, $R C P$, résonna pendant des décennies comme un label de scientificité publique, les deux termes étant alors indissociables, confondus, en rupture avec la période présente dans laquelle, par exemple, I'Agence nationale de la recherche (ANR) qui convoque aussi I'interdisciplinarité, est une instance privée, qui n'est pas astreinte dans ses financements aux réglementations de la recherche étatique.

Dans ces dispositifs pluridisciplinaires d'antan, la posture de l'ethnologie - à travers ceux qui tiennent toujours à se nommer ethnographes - attirera tout d'abord l'attention du lecteur, tant elle s'inscrit en décalage avec les autres disciplines. Alors que les sociologues, dans la foulée de Mendras, repèrent tous les signes de transformations sociales majeures et décrivent la fin des campagnes repliées sur elles-mêmes, les ethnologues s'attachent au contraire à reconstruire dans l'imaginaire des univers traditionnels figés, éloignant de leur vision tout ce qui pourrait corrompre les vestiges qu'ils s'efforcent de pérenniser Les personnages sont sculpturalisés, les anciennes techniques auréolées de savoirfaire aussi extraordinaires qu'immuables, les structures sociales rigidifiées. Exit les migrations qui ont débuté au début du $x x^{e}$ siècle et $s$ accélèrent après la Seconde Guerre mondiale. Le politique est tout autant banni de ce champ ethnographique magnifié, où les équilibres régneraient tout comme des complémenta- 
rités organiques. L'ethnologue ne reconnaît ainsi aucun rapport de domination et surtout pas entre hommes et femmes unis par l'accomplissement rayonnant de leurs tâches, rôles et devoirs. Le lecteur ne sera pas surpris que des frictions entre disciplines aient surgi et ce d'autant plus que certains ethnologues qui contribuent aujourd'hui à l'ouvrage semblent n'avoir toujours pas compris les enjeux fondamentaux de ces visions contradictoires. Tandis qu'Edgar Morin auprès des jeunes et des femmes de Plozevet cherchait à penser le changement social, les ethnologues croyant garantir leur légitimité disciplinaire, tentaient de transférer de façon mimétique l'ethnographie coloniale que les indépendances avaient chassée dix ans avant, avec dans ses valises leurs collaborateurs scientifiques. Corollairement à l'exception de l'un d'entre eux, ces ethnologues avouent avec ingénuité n'avoir pas entrevu de bouleversement dans leur propre microcosme scientifique que 1968 n'aurait pas atteint, pas plus que par ailleurs le féminisme dont ils n'avaient pas entendu parler. Qualifiés de folkloristes à juste titre, ces ethnologues producteurs de chimères grâce à ceux qu'ils appellent " nos informateurs " comme dans les colonies qui leur servent de modèles, ne rencontrent pas plus de succès auprès des populations dont ils ont façonné l'image. Pour des raisons antithétiques qu'à Plozevet où E. Morin met en avant les contestataires, ce qui n'est localement guère apprécié, les relations avec ceux avec qui les ethnologues n'ont pas dialogué sont rompues car ils se perçoivent enfermés dans une " arriération " stigmatisante. Lorsqu'elles sont exceptionnellement maintenues, le caractère anecdotique des fragments de méditation, après plus de trente ans de terrain, interpelle le lecteur qui est invité à se pénétrer du dogme ethnographique d'une vie dédiée à un seul Autre dont on ne saurait se séparer. Pour la mémoire rappelons qu'à la même période une anthropologie différente avec ses facettes plurielles existait en dépit de la marginalisation où la refoulaient des îlots institutionnels dits académiques. En opposition à ce conservatisme dérisoire et anachronique, Balandier présentait une " sociologie dynamique " pour éviter d'être assimilé à l'ethnographie coloniale, Althabe avançait une anthropologie politique, Jaulin de son côté dirigeait le plus grand département d'ethnologie à l'université de Paris VII où se coagulaient en communauté de pensée 500 étudiants. Enfin, l'anthropologie urbaine voyait ses premières investigations s'attaquer aux ZUP et cités HLM réputées anonymes alors que les ethnographes des enquêtes pluridisciplinaires édifiaient de fermes résistances à tout ce qui pouvait évoquer une modernisation de leurs chères campagnes françaises qu'il fallait sauvegarder d'urgence, au moins en pensée.

L'admirable entretien que Bernard Paillard réalise avec E. Morin permet de comprendre les fossés qui séparaient donc de toutes les autres disciplines de sciences sociales, tournées vers un déchiffrage du présent, l'ethnologie isolée dans un rôle absurde de gardienne d'un temple d'illusions. Dans ce tableau, la Corse tient une place à part tant la politisation et l'idéologisation du terrain ont obligé l'ethnologue à une réflexivité aiguë sur ses propres inclinaisons et les implications de ses recherches.

Soulignons pour conclure que l'ensemble des contributions qui ciblent sous des angles très divers Plozevet - et aussi les autres enquêtes - illustrent de façon très concrète et stimulante les modes de production des connaissances avec leurs parts idéelles, interpersonnelles, sociales, économiques, politiques. Les relations hiérarchiques, les statuts internes à l'en- 
quête, le jeu des origines de classe et des appartenances sexuées sont mis en scène avec toute leur pesanteur et leur efficacité symbolique, y compris ou surtout lorsqu'elles n'affleurent pas encore à la conscience de leurs auteurs tant d'années après. Ainsi en va-t-il de ces chercheurs masculins qui « installent leur famille " - soit épouse et enfants - sur leur terrain, libres ainsi de vaquer à leurs nobles tâches d'investigation. Comment auraient-ils pu dans ces conditions entrevoir que les migrations féminines rurales vers la ville prenaient leur sens dans un désir de liberté des femmes souhaitant échapper aux rapports d'assignation et de domination internes à leurs collectivités familiales et microsociales ?
Remercions donc les coordinateurs de l'ouvrage de livrer, sous sa forme brute, non polissée, pourrait-on dire " authentique ", une matière aussi riche pour l'épistémologue des sciences sociales, qui souhaite aller au-delà des modes actuelles qui mettent au pinacle « l'arrière-cour » de la recherche mais de fait favorisent le narcissisme du chercheur aux dépens de la rigueur analytique. S'il est un défaut dont les chercheurs des enquêtes pluridisciplinaires de cette époque sont dépourvus, c'est bien l'égotisme, tant ils sont pour la plupart dédiés à une objectivité scientifique mythifiée, supposant la mise à distance des sujets singuliers qui construisent la société.

Monique SELIM

Institut de recherche pour le développement (IRD) UMR 201 - Paris 1 